\title{
Reliability of a radiation-free, noninvasive and computer-assisted assessment of the spine in children with cerebral palsy
}

\author{
Kristina Ruthard ${ }^{1} \cdot$ Anke Raabe-Oetker $^{1} \cdot$ Johannes Ruthard $^{2} \cdot$ Thomas Oppermann $^{3} \cdot$ Ibrahim Duran $^{3}$ (1) . \\ Eckhard Schönau ${ }^{4}$
}

Received: 17 July 2019 / Revised: 14 November 2019 / Accepted: 30 January 2020 / Published online: 8 February 2020

(C) The Author(s) 2020

\begin{abstract}
Purpose The radiation-free, noninvasive and computer-assisted Spinal Mouse ${ }^{\circledR}(\mathrm{SM})$ is a reliable and valid measuring instrument for functional analysis of the pediatric spine. The aim of this study was to examine the intra-rater reliability of the SM measurements in children with cerebral palsy $(\mathrm{CP})$ and to investigate differences after a 1 week of the rehabilitation program. Methods A total of $168 \mathrm{SM}$ investigations in the sagittal plane and frontal plane at three measurement times from a sample of 28 children ( $n=10$ girls, age $9.7 \pm 3.1$ years) with CP were eligible for evaluation. For the verification of reliability, the measurement results from the first and second measurement times $(t 1, t 2)$ were used at intervals of 1 day. In addition, differences after the rehabilitation program the patients underwent $(t 3)$ were evaluated using the measurement results of the first and third measurements (5-day interval).

Results The results show good to excellent intra-rater reliability for the SM measurements, both in the sagittal and in the frontal plane (ICC values $=0.69-0.99$ ). Furthermore, significant changes may occur after only 1 week of therapeutic intervention for total spinal inclination $(t 1: 12.82 \pm 5.40, t 3: 11.11 \pm 5.60, p=0.014$, Cohen's $d=0.43)$ and spine length $(t 1$ : $401.75 \pm 69.05, t 3: 409.25 \pm 63.58, p=0.030$, Cohen's $d=0.43$ ).

Conclusions SM can be used to generate reliable values for functional analysis of the spine in children with CP. Furthermore, significant posture differences can be demonstrated by therapeutic interventions, especially in the spine inclination (Inc) and spine length (SL).
\end{abstract}

\section{Graphic abstract}

These slides can be retrieved under Electronic Supplementary Material.
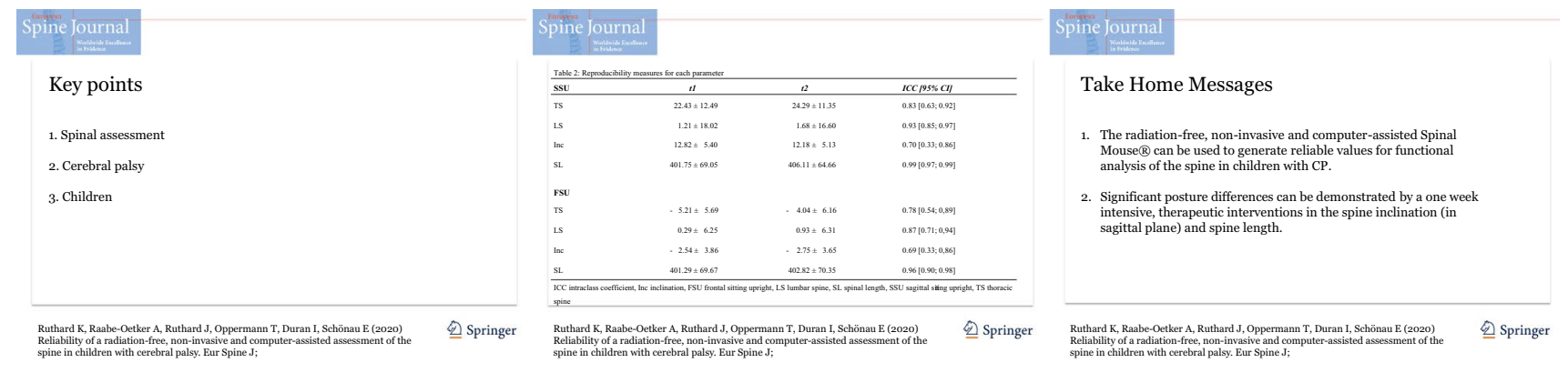

Keywords Spinal assessment $\cdot$ Cerebral palsy $\cdot$ Reliability $\cdot$ Spinal Mouse $\cdot$ Children

Ibrahim Duran and Eckhard Schönau have shared last authorship.

Electronic supplementary material The online version of this article (https://doi.org/10.1007/s00586-020-06328-4) contains supplementary material, which is available to authorized users.

Extended author information available on the last page of the article
Abbreviations
CP Cerebral palsy
FSU Frontal sitting upright
INC Spine inclination
LS Lumbar spine
SL Spine length 
SM Spinal Mouse

SSU Sagittal sitting upright

TS Thoracic spine

\section{Introduction}

Cerebral palsy (CP) is a group of disorders that result from non-progressive damage to the developing brain [1]. With an incidence of 2-3 per 1000 live births, it is the most common cause of childhood and adolescent movement disorder [2]. Approximately a quarter of the children and adolescents with CP develop neuromuscular scoliosis during their lifetime and suffer from spinal deformity [3, 4]. Over time, the deformity can lead to pain, as well as complicating the motor, pulmonary and cardiac situation due to the lateral bending of the costal arch toward the iliac crest [5, 6]. Early detection of scoliosis and severity, measured by the Cobb angle, is essential to start therapy, such as physiotherapy (PT) and brace treatment [7, 8]. In order to determine the Cobb angle, those affected regularly undergo radiographic $\mathrm{X}$-ray examination since childhood $[9,10]$. This screening contributes to the increased radiation exposure of this patient population (e.g., due to the radiographic screening for hip dislocation).

A noninvasive, radiation-free and analog-digital measuring device for measuring the spine surface is the Spinal Mouse (SM). The SM measurement is a computer-assisted measurement method that analyzes and displays the malpositions, postural weaknesses and mobility of individual segments of the spine in the sagittal plane (erection, flexion and extension) as well as in the frontal plane (erection and lateral flexion to the left and right). Scientific publications have already demonstrated the validity and reliability of use in healthy children and adults $[4,11,12]$. The validity of the SM was determined by comparing the results of the lumbar segment mobility of X-ray examinations and the SM [4]. Although people who suffer from CP need regular spinal examinations, there are no studies with SM.

Thus, the primary aim of this study was to investigate the intra-rater reliability of SM in children and adolescents with $\mathrm{CP}$. The second aim was to evaluate the possible application in the rehabilitation program of children with $\mathrm{CP}$.

\section{Method}

\section{Study population}

The study population consists of children with $\mathrm{CP}$, who participated in the rehabilitation program "Auf die Beine" at the Center for Prevention and Rehabilitation of the University Hospital of Cologne from November 2017 to January 2018.
The rehabilitation program "Auf die Beine" had already been presented in detail in a previous publication [13]. Overall, 276 investigations were performed in 46 children with CP (Gross Motor Function Classification System (GMFCS) I-IV) in the studied period.

The GMFCS was used to define the level of motor function of the children with CP. This well-established tool consists of a 5-point ordinal scale, designated as I (best motor function) to $\mathrm{V}$ (worst motor function) [14]. Children with $\mathrm{CP}$, GMFCS level I and II, are free walkers, while children with CP, GMFCS level III-V, need additional support, or are unable to walk.

Only measurements in children with GMFCS stages I, II and III were further evaluated ( 39 children). After applying the additional exclusion criteria (1 not complete investigation, 3 not independent implementation, 3 stayed longer in therapy, 4 not understand instruction), another 11 children were excluded. Totally, 168 investigations with one measurement each in the sagittal and frontal plane with three consecutive SM assessments ( $t 1-t 3)$, from a sample of 28 children, were eligible for examining the intra-rater reliability (Fig. 1). The measurements took place on three different days - $t 1$ (Monday), $t 2$ (Tuesday) and $t 3$ (Friday). The physical characteristics of the participants are given in Table 1.

The guardians of the respective participant and the participants who were able had to sign the permission. The study was approved by the Ethics Committee of the University of Cologne (No. 17-326). A detailed description of the registry can be found at http://www.germanctr.de (DRKS00013759) which is a primary register of the International Clinical Trials Registry Platform of the World Health Organization.

\section{Spinal Mouse, investigation and variables}

The investigations were conducted using the measuring system SM of the company idiag (Nr.: DM/043 705, idiag AG, Fehraltorf, Switzerland) with the Software Version 1.14.0.0, which is a class 1 medical device (Fig. 2). On the bottom is a big wheel, which records the length of the spine, and a potentiometer, which measures the angles relative to the vertical at a rate of $150 \mathrm{~Hz}$ [12]. Due to this type of measuring device, the angular position of the individual vertebrae can be performed in almost any body position.

For all measurements, the children had to clear their upper body and sit on a chair. At the beginning, the examiner marked the start and end points (third cervical vertebra and anus fold). Through a standardized movement task, the children were asked by the examiner to take certain measurement positions. In total, two measurements were made, one in the sagittal plane (sagittal sitting upright $=$ SSU) and one in the frontal plane (frontal sitting upright $=$ FSU). The following variables, which were also used in other studies for comparison [11, 15], were assessed for spinal evaluation: 


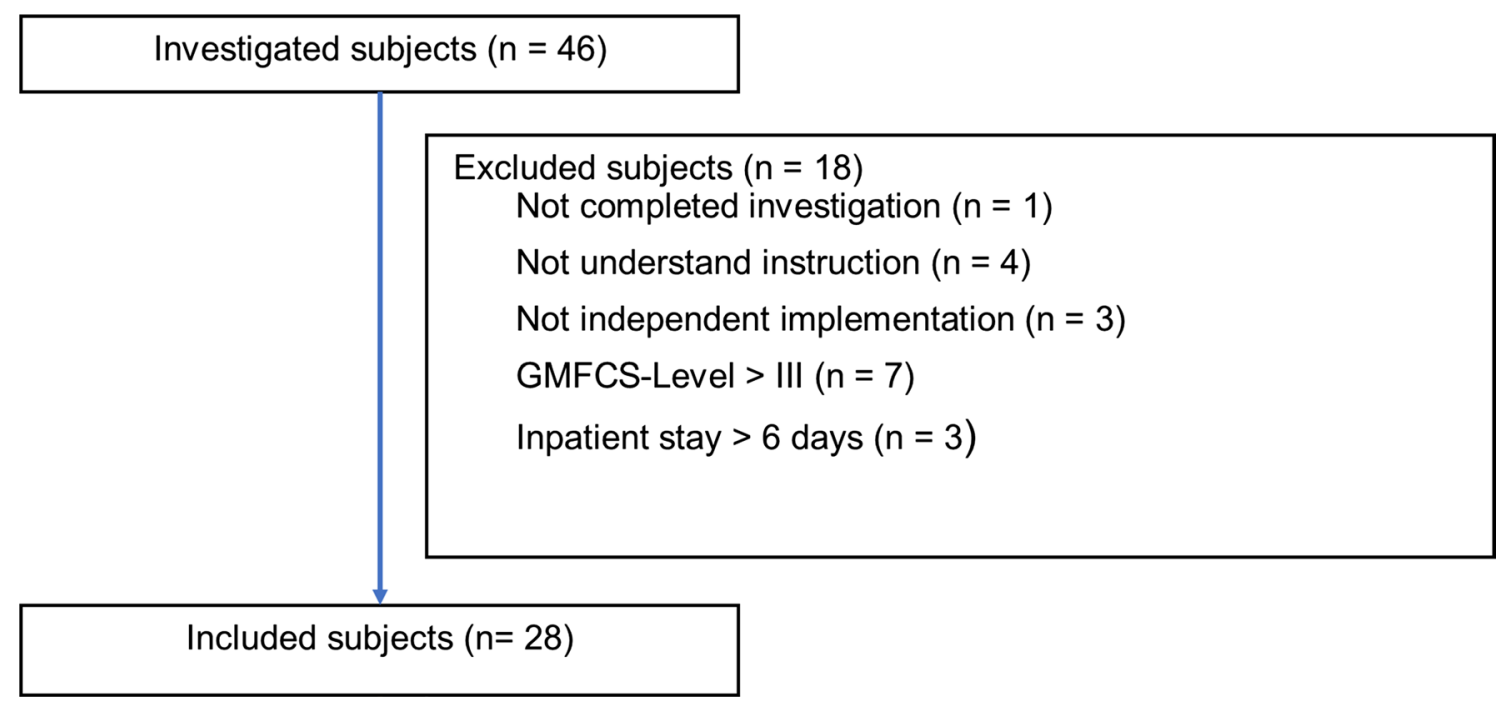

Fig. 1 Flowchart on sample selection and criteria

Table 1 Study population

\begin{tabular}{lc}
\hline Variables & Total \\
\hline Participants $(n)$ & $28(100)$ \\
Female $(n)$ & $10(35.7)$ \\
Age (years) & $9.7(3.1)$ \\
Height $(\mathrm{cm})$ & $128.9(19.8)$ \\
Weight $(\mathrm{kg})$ & $29.9(14.9)$ \\
GMFCS level $(n)$ & \\
I & $2(7.1)$ \\
II & $10(35.7)$ \\
III & $16(57.1)$ \\
CP subtype $(n)$ & \\
Bilateral spastic & $21(75.0)$ \\
Unilateral spastic & $2(7.1)$ \\
Dyskinetic & $1(3.6)$ \\
Ataxic & $2(7.1)$ \\
Mixed type & $2(7.1)$ \\
\hline
\end{tabular}

Data presented as mean (SD) or $n(\%)$

thoracic spine (TS), lumbar spine (LS), inclination (Inc) and spine length (SL). The TS, LS as well as the Inc are regional angles that result from the sum of the individual segment angles and are therefore given in angular degrees. The SL is given in millimeters.

\section{Intra-rater reliability and therapeutic effect}

Overall, the subjects were examined within 1 week at three measuring times (Monday, Tuesday and Friday) with the SM. Two measurements were taken at the time of each measurement so that a total of 168 measurements were

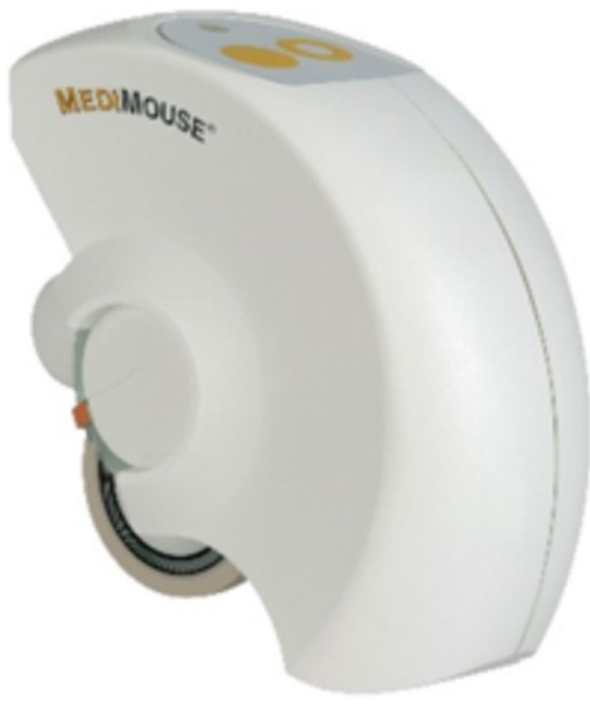

Fig. 2 Spinal Mouse in lateral view

available for evaluation at the end of the study. To check the intra-rater reliability, the measurements from the first $(t 1)$ and second measurement times $(t 2)$ were used. Furthermore, the clinical application to evaluate the therapeutic effect of 1-week intensive rehabilitation program on the spine of children with CP was examined in the study. During the 1-week treatment, the children were given every day $4 \mathrm{~h}$ of goal-oriented PT in combination with vibration-assisted PT. It was examined whether after 1 week of therapeutic treatments already an improved spinal column erection is to be measured. For this analysis, only measurements in the sagittal plane from the first $(t 1)$ and third 
measurement times $(t 3)$ were used since the clinical experience so far, especially the trunk erection of the children improved under the rehabilitation program.

\section{Statistical analysis}

We used the intraclass correlation coefficient (ICC) to assess the inter-day and intra-rater reliability of the SM. According to McGraw and Wong [16], the ICC $(\mathrm{C}, 3)$ was used for an adjusted (consistency) two-way random model for single values. According to Livanelioglu [17], ICC values between 0.60 and 0.74 reveal a good reproducibility and ICC values $\geq 0.75$ an excellent reproducibility, respectively. All the collected data were first checked for normal distribution. If these were present, the change in the mean values of the before-after comparison with the dependent $t$ test was checked for significance. For results that did not show normal distribution, the Wilcoxon test was used. By means of these tests, it was examined whether there were significant changes in the mean values during the 1-week therapeutic intervention. Cohen's d was used to calculate the effect size [18]. The significance level was $<0.05$ for italic values.

To check whether the age or the sex has an influence on the measurement results, a variance analysis (without repeat measurement) was performed for the normally distributed variables. The Kruskal-Wallis test was used for the non-normal distributed values. Statistical analyses were conducted using the software SPSS Statistics Version 25 (SPSS Inc., Illinois, USA).

\section{Results}

\section{Study population}

The participants' demographics are given in Table 1. The mean age of the children included for investigation was $9.7 \pm 3.1$ years of age (Table 1). Among the investigated children, $35.7 \%$ were girls. Only the spine length differed with increasing age (SSU-SL Pearson's $r=0.848, p<0.001$; FSU-SL Pearson's $r=0.856, p<0.001)$. No statistically significant relation of age and gender could be shown with TS, LS and INC (in SSU and FSU, $p=0.069-0.966$ ).

\section{Intra-rater reliability}

The calculations of the ICC for all variables showed values between 0.69 and 0.99 in the sagittal plane and in the frontal plane (Table 2). Chiefly, the spine length showed ICC values between 0.96 and 0.99 in both measuring positions.

Furthermore, the ICC values were differentiated relative to the GMFCS level to see if there were any differences in the reproducibility by the severity of the CP. Since the group
Table 2 Reproducibility measures for each parameter

\begin{tabular}{cccc}
\hline & \multicolumn{2}{c}{$t 2$} & ICC $[95 \% \mathrm{CI}]$ \\
\hline SSU & & & \\
TS & $22.43 \pm 12.49$ & $24.29 \pm 11.35$ & $0.83[0.63 ; 0.92]$ \\
LS & $1.21 \pm 18.02$ & $1.68 \pm 16.60$ & $0.93[0.85 ; 0.97]$ \\
Inc & $12.82 \pm 5.40$ & $12.18 \pm 5.13$ & $0.70[0.33 ; 0.86]$ \\
SL & $401.75 \pm 69.05$ & $406.11 \pm 64.66$ & $0.99[0.97 ; 0.99]$ \\
FSU & & & \\
TS & $-5.21 \pm 5.69$ & $-4.04 \pm 6.16$ & $0.78[0.54 ; 0,89]$ \\
LS & $0.29 \pm 6.25$ & $0.93 \pm 6.31$ & $0.87[0.71 ; 0,94]$ \\
Inc & $-2.54 \pm 3.86$ & $-2.75 \pm 3.65$ & $0.69[0.33 ; 0,86]$ \\
SL & $401.29 \pm 69.67$ & $402.82 \pm 70.35$ & $0.96[0.90 ; 0.98]$ \\
\hline
\end{tabular}

$I C C$ intraclass coefficient, Inc inclination, FSU frontal sitting upright, $L S$ lumbar spine, $S L$ spinal length, $S S U$ sagittal sitting upright, $T S$ thoracic spine

of probands with a GMFCS level I demonstrated only two subjects, the groups of subjects with a GMFCS level I and II were summarized as the "mild" CP group. The analysis showed no significant differences between children with mild and moderate CP (GMFCS III).

\section{Therapy effect}

The variables SL $(t 1: 401.75, t 3: 409.25, p=0.014)$ and Inc $(t 1: 12.82, t 3: 11.11, p=0.030)$ were in the measuring position SSU significant differences between the first and the third measuring time. The effect size of the SL according to Cohen's d was $d=0.43$ and corresponded to a small to medium effect size, respectively, and Cohen's $d=0.43$ is also of the variable Inc.

\section{Discussion}

In this study, we evaluated the inter-day and intra-rater reliability of the SM in children with CP. The measurements were taken in the sagittal plane and in the frontal plane using the SM (idiag AG, Switzerland). By calculating the ICC for the two measurement times $t 1$ and $t 2$, values of 0.69-0.99 could be determined (Table 2). For all ICC calculations of the parameters, six values were above 0.75 . Based on the study by Livanelioglu and Kellis [11, 17], in which SM was also used as a measuring instrument for scoliosis research in boys and in healthy boys, the available ICC scores can be described as excellent. In the study of Kellis, they found ICCs ranged from 0.61 to 0.96 . Only two parameters of our study show ICC values between 0.69 and 0.75 . These results are also reliable to good ICC values [17]. In addition, there was no significant difference in the inter-day and intra-rater reliabilities between children with mild CP (GMFCS level 
Table 3 Effect of the rehabilitation program on the measured variables of the Spinal Mouse

\begin{tabular}{ccccl}
\hline & $t 1$ & $t 3$ & $p$ value & Cohen's $d$ \\
\hline SSU & & & & \\
TS & $22.34 \pm 12.47$ & $22.18 \pm 10.20$ & 0.881 & 0.02 \\
$L S$ & $1.21 \pm 18.02$ & $-0.68 \pm 19.03$ & 0.395 & 0.20 \\
Inc & $12.82 \pm 5.40$ & $11.11 \pm 5.60$ & 0.030 & 0.43 \\
SL & $401.75 \pm 69.05$ & $409.25 \pm 63.58$ & 0.014 & 0.43 \\
\hline
\end{tabular}

Inc inclination, $S L$ spinal length, $S S U$ sagittal sitting upright

I-II) and moderate CP (GMFCS level III). During the 276 assessments with the SM, no adverse effect had occurred.

To evaluate the therapy effect, the data of the first and the third measuring time were used for calculation. After the 1-week intervention through the rehabilitation program, significant changes in the measurement of SSU for the Inc and the SL could be shown (Table 3). Both parameters show a change within the 1-week therapy. The inclination of the total vertebral column is calculated from the perpendicular of the imaginary line by the vertebrae $\mathrm{C} 7$ and $\mathrm{S} 3$ - start and end points of the measurement. Children with healthy backs ideally have an inclinatory value of almost 0 in the position SSU. A significantly smaller value of the inclination at the time of measurement $t 3$ in an upright sitting position makes it clear that the subjects were able to assume a more vertical sitting position after 1 week of therapy. For this change, an effect strength of Cohen's $d=0.43$ could be calculated, which corresponds to a small to medium effect.

The improved spine erection is confirmed with the analyzed SL value. At the SL, the measured values of $t 3$ show significantly larger values than at $t 1$. For this parameter, larger readings correspond to a longer spine. The values show that the spine has extended on average by almost $1 \mathrm{~cm}$. A length growth of the spine within a week is to be excluded in this case. It is believed that the improved erection after therapeutic intervention in a sagittal seated position made it possible for the children to be stretched more extensively and thus the vertebral bodies stood more vertically on top of each other. A positive therapeutic effect of this rehabilitation program on the gross motor function (assessed by gross motor function measure) of children with $\mathrm{CP}$ was already reported [19].

\section{Limitations}

The study population consisted of participants in the rehabilitation program "Auf die Beine." Although the authors did not identify any selection bias, it can still not be excluded.

In addition, the dates for the SM examination had to be subordinate to the time frame of the rehabilitation program.
As a result, the subjects had different therapy applications before the measurements and it often happened that the subjects were already very tired from the previous therapy. Accordingly, the general physical condition also had an impact on compliance. A further limitation is the understanding of the task of movement. Children with $\mathrm{CP}$ have different cognitive prerequisites, making the standardized test procedure more difficult. It is recommended to conduct further studies with a larger study population.

The period ( 1 week) used in this study to evaluate the effect of the rehabilitation program on posture is too short to assess effects on scoliosis.

\section{Conclusion}

In the present study, it was shown that a functional analysis of the spine with SM is feasible and safe in children with $\mathrm{CP}$ and that the measurement results show a good to excellent inter-day and intra-rater reliability. Since the SM is a radiation-free and noninvasive measurement system, multiple measurements over time to monitor the development of the spine in children with $\mathrm{CP}$ are possible. Therefore, the monitoring of the spine in children with CP with the SM might be a useful supplement to any necessary $\mathrm{X}$-ray examinations.

Acknowledgements Open Access funding provided by Projekt DEAL.

Funding This research received no specific grant from any funding agency in the public, commercial or not-for-profit sectors.

\section{Compliance with ethical standards}

Conflict of interest The authors declare that they have no conflict of interest.

Open Access This article is licensed under a Creative Commons Attribution 4.0 International License, which permits use, sharing, adaptation, distribution and reproduction in any medium or format, as long as you give appropriate credit to the original author(s) and the source, provide a link to the Creative Commons licence, and indicate if changes were made. The images or other third party material in this article are included in the article's Creative Commons licence, unless indicated otherwise in a credit line to the material. If material is not included in the article's Creative Commons licence and your intended use is not permitted by statutory regulation or exceeds the permitted use, you will need to obtain permission directly from the copyright holder. To view a copy of this licence, visit http://creativecommons.org/licenses/by/4.0/.

\section{References}

1. Bax M, Goldstein M, Rosenbaum P et al (2005) Proposed definition and classification of cerebral palsy. Dev Med Child Neurol 47(8):571-576 
2. Cans C (2000) Surveillance of cerebral palsy in Europe: a collaboration of cerebral palsy surveys and registers. Surveillance of Cerebral Palsy in Europe (SCPE). Dev Med Child Neurol 42(12):816-824

3. Koop SE (2009) Scoliosis in cerebral palsy. Dev Med Child Neurol 51(Suppl 4):92-98

4. Guermazi M, Ghroubi S, Kassis M et al (2006) Validité et reproductibilité du Spinal Mouse pour l'étude de la mobilité en flexion du rachis lombaire. Annales de readaptation et de medecine physique: revue scientifique de la Societe francaise de reeducation fonctionnelle de readaptation et de medecine physique 49(4):172-177

5. Persson-Bunke M, Hägglund G, Lauge-Pedersen H et al (2012) Scoliosis in a total population of children with cerebral palsy. Spine 37(12):E708-E713

6. Murphy RF, Mooney JF (2019) Current concepts in neuromuscular scoliosis. Curr Rev Musculoskelet Med. https://doi. org/10.1007/s12178-019-09552-8

7. Döderlein L (2015) Infantile zerebralparese: diagnostik, konservative und operative therapie, vol 2. Springer, Berlin

8. Tsirikos AI (2010) Development and treatment of spinal deformity in patients with cerebral palsy. Indian J Orthop 44(2):148-158. https://doi.org/10.4103/0019-5413.62052

9. Trobisch P, Suess O, Schwab F (2010) Idiopathic scoliosis. Dtsch Arztebl Int 107(49):875-83; quiz 884. https://doi.org/10.3238/ arztebl.2010.0875

10. Khodaei M, Pachêco-Pereira C, Trac S et al (2018) Radiographic methods to estimate surgical outcomes based on spinal flexibility assessment in patients who have adolescent idiopathic scoliosis: a systematic review. Spine J 18(11):2128-2139. https://doi. org/10.1016/j.spinee.2018.06.344

11. Kellis E, Adamou G, Tzilios G et al (2008) Reliability of spinal range of motion in healthy boys using a skin-surface device. J Manipulative Physiol Ther 31(8):570-576. https://doi. org/10.1016/j.jmpt.2008.09.001

12. Mannion AF, Knecht K, Balaban G et al (2004) A new skin-surface device for measuring the curvature and global and segmental ranges of motion of the spine: reliability of measurements and comparison with data reviewed from the literature. Eur Spine J Off Publ Eur Spine Soc Eur Spinal Deform Soc Eur Sect Cerv Spine Res Soc 13(2):122-136

13. Stark C, Hoyer-Kuhn H-K, Semler O et al (2015) Neuromuscular training based on whole body vibration in children with spina bifida: a retrospective analysis of a new physiotherapy treatment program. Childs Nerv Syst 31(2):301-309. https://doi. org/10.1007/s00381-014-2577-2

14. Palisano R, Rosenbaum $P$, Walter $S$ et al (1997) Development and reliability of a system to classify gross motor function in children with cerebral palsy. Dev Med Child Neurol 39(4):214-223

15. Kavruk H (2010) Der Einfluss des Tragens von Säuglingen und Kleinkindern in Tragehilfen auf die Entwicklung von Haltungsschäden im Schulkindalter: Untersuchungen mit der MediMouse, Köln

16. McGraw KO (1996) Forming inferences about some intraclass correlation coefficients. American Psychological Association, Washington, D.C.

17. Livanelioglu A, Kaya F, Nabiyev V et al (2016) The validity and reliability of "Spinal Mouse" assessment of spinal curvatures in the frontal plane in pediatric adolescent idiopathic thoraco-lumbar curves. Eur Spine J Off Publ Eur Spine Soc Eur Spinal Deform Soc Eur Sect Cerv Spine Res Soc 25(2):476-482. https://doi. org/10.1007/s00586-015-3945-7

18. Cohen J (1988) Statistical power analysis for the behavioral sciences, 2nd edn. Erlbaum, Hillsdale

19. Duran I, Stark C, Martakis K et al (2019) Reference centiles for the gross motor function measure and identification of therapeutic effects in children with cerebral palsy. J Eval Clin Pract 25(1):78 87. https://doi.org/10.1111/jep.12990

Publisher's Note Springer Nature remains neutral with regard to jurisdictional claims in published maps and institutional affiliations.

\section{Affiliations}

\section{Kristina Ruthard ${ }^{1} \cdot$ Anke Raabe-Oetker $^{1} \cdot$ Johannes Ruthard $^{2} \cdot$ Thomas Oppermann $^{3} \cdot$ Ibrahim Duran $^{3}$ (D) Eckhard Schönau ${ }^{4}$}

Ibrahim Duran

Ibrahim.duran@unireha-koeln.de

1 Institute for Exercise Therapy and Movement-Oriented Prevention and Rehabilitation - Dept. 2, German Sport University, Cologne, Germany

2 Medical Faculty and University Hospital, Institute for Clinical Chemistry, University of Cologne, Cologne, Germany
3 Medical Faculty and University Hospital, Center of Prevention and Rehabilitation, UniReha, University of Cologne, Lindenburger Allee 44, 50931 Cologne, Germany

4 Department of Pediatrics, Medical Faculty and University Hospital, University of Cologne, Cologne, Germany 